\title{
Detection of calcium in rice using Laser-Induced Breakdown Spectroscopy (LIBS)
}

\author{
Rahmat Abdul Wahid*, Asiah Yahaya and Yusof Munajat \\ Department of Physics, Faculty of Science, Universiti Teknologi Malaysia, UTM Skudai, 81310, Johor, Malaysia. \\ *Corresponding Author: rahmatabdulwahid@gmail.com (R.A. Wahid)
}

\section{Article history :}

Received 1 March 2014

Revised 12 May 2014

Accepted 19 May 2014

Available online 1 June 2014

\section{GRAPHICAL ABSTRACT}

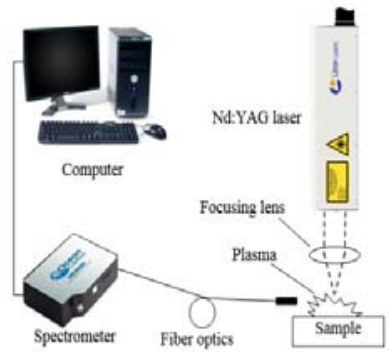

\begin{abstract}
Laser-Induced Breakdown Spectroscopy (LIBS) is a very reliable technique for both qualitative and quantitative analyses. Detection of elements composition is important to help consumer choose the right variety of rice. In this paper, an ungated LIBS system was used to detect calcium in rice. An experimental setup was designed using Nd:YAG laser operating at $1064 \mathrm{~nm}(60 \mathrm{~mJ}$ per pulse) and a spectrometer connected to a fiber optic in order to collect the atomic emission which is displayed on a computer. Twelve samples of rice were purchased from local market which offered differences with regard to their rice varieties, grain size and polishing rate. The calcium concentration of each sample was initially evaluated by using Atomic Absorption Spectroscopy (AAS) after acid digestion. Pellets of approximately $5 \mathrm{~mm}$ thick and $40 \mathrm{~mm}$ diameter were prepared by transferring $10 \mathrm{~g}$ of ground rice sample to a $40 \mathrm{~mm}$ die set and applying $40 \mathrm{MPa}$. For the LIBS analysis, Nd:YAG laser was used to ablate the surface of each pellets. Fifty spectra from different spots of the pellets were collected by the spectrometer from each sample. The spectra were then averaged and analyzed using OriginPro 8.5 for baseline removal and peak findings. Two calcium (Calcium II) lines with high intensity were observed at the wavelength of 393.37 $\mathrm{nm}$ and $396.85 \mathrm{~nm}$. The study shows that LIBS can be an excellent tool for detecting calcium in rice.
\end{abstract}

Keywords: Laser-Induced Breakdown Spectroscopy (LIBS), Rice pellet, Calcium detection

(C) 2014 Penerbit UTM Press. All rights reserved http://dx.doi.org/10.11113/mjfas.v10n2.59

\section{INTRODUCTION}

Rice (Oryza sativa) is a staple food among Malaysian. A staple food is a food that is consumed regularly and in a large amount of a person diet. It supplies a major proportion of energy and nutrient needs [1]. Rice is most commonly eaten as cooked entire grains, but it is also milled into flour or meal which is used to make noodles and porridges. Rice contains valuable minerals such as calcium, iron, magnesium, phosphorus, potassium, sodium, zinc, copper, manganese and selenium. These minerals in rice are important for human especially calcium which can be found in a large amount compared to other minerals [2].

Calcium is the fifth most abundant element in human's body with $99 \%$ of it occurring in bone and teeth. Only one percent of calcium in human body is located outside of the bone. On a dry weight basis, bone contains about $150 \mathrm{mg}$ calcium/gram bone. On comparison, soft tissue such as liver, muscle or brain contains less than 35 $\mu \mathrm{g}$ calcium/gram tissue. It is essential for normal blood clotting, muscle contraction, membrane permeability, myocardial function and normal neuromuscular excitability. Inadequate calcium intake signs include bone calcification and growth in children (rickets) and weak bones in adults (osteoporosis) [3].
Calcium is important in human's diet, data available from several general dietary studies among Malaysians of different age categories often show low calcium intakes below $500 \mathrm{mg}$ per day that may lead to osteoporosis. Studies found that the main calcium sources for Malaysians were from vegetables and bean. It is hard to change ones dietary intake, thus enriching the existing food with calcium can be the best solution [4]. Since rice is a food staple among Malaysian, it is possible to enriched or in other word, rice grain biofortification with calcium hence becoming a high-calcium food source. Although not particularly rich in calcium, rice consumed in large quantities can substantially increase the dietary calcium content $[1,5]$. In this sense, determining the calcium amount in packed rice is important in order to choose the right rice with balanced nutrient.

There were four main attributes of rice that have been studied by local researchers which were done in thirteen cities in Malaysia. Results indicated that food safety was the most important attribute for rice followed by taste, size of grain and price. The findings suggested that Malaysian consumers have a health and safety perspective for choosing the right variety of rice for them. Thus labelling the packet of rice with nutritional contents, chemicals used and method of production also 
could be very useful in order to give freedom to consumer to choose their preferred rice [6].

Conventional calcium analysis methods consist of these steps: taking a sample from the material or from a processing line product, transfer it to an inspection device or to a laboratory, preparing it for chemical analysis and for determination. The sample preparation for chemical analysis is a compulsory stage for many analytical techniques, which consists in transforming the sample into an appropriate form for the analysis. For the elemental analysis using this technique, the solid samples are usually digested using aqua regia to solutions, which carry the analytes free of organic matter. This step usually takes a lot of time for analytical procedure; it can also produces toxic residues and increases the probability of sample contamination [7].

Laser-Induced Breakdown Spectroscopy (LIBS) is a method of atomic emission spectroscopy (AES) that uses laser-generated plasma as the hot vaporization, atomization and excitation source. LIBS have the ability to interrogate samples in situ and remotely without any preparation. Qualitative and quantitative analyses are carried out by monitoring emission line positions and intensities. LIBS system mainly consists of a pulsed laser, optics, a delay generator and a spectrometer [8]. For the solid sample analysis, high-energy pulse laser is focused on the surface of the sample. The pulsed laser emits a burst of coherent electromagnetic radiation. When it is incident on a surface, some of the incident energy is absorbed and converted into heat and the remainder is reradiated back as the reflected pulse or scatters from the surface. The laser at given a power density will cause the particles at the surface to be ablated. The ablated material dissociates into excited ionic and atomic species. Collection and examination of the emitted light provides the analysis because each element has a unique emission spectrum useful to represent the species $[8,9]$. Example of collection of the emitted light is shown in Figure 1, a different peak represents different elements, while the intensity of the peak may represents its concentration.

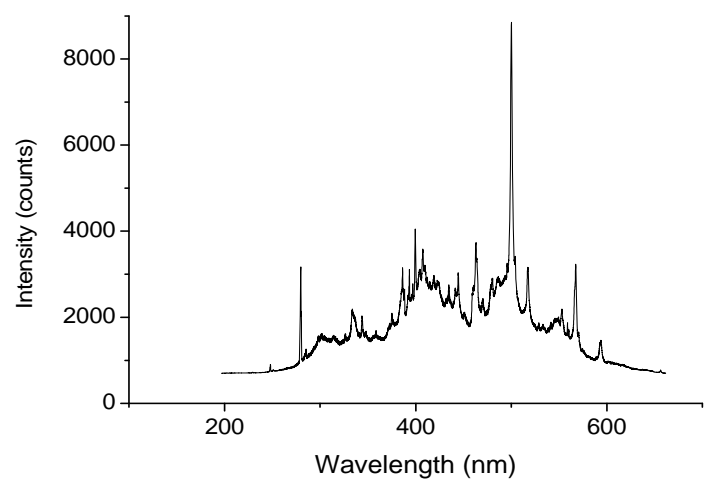

Fig. 1 LIBS spectrum of rice from near ultraviolet until near infrared region spectrometer. Any of these lines could potentially be useful for detection of minerals in rice.
LIBS like other analytical methods displays matrix effects which will make quantitative analysis more difficult to be executed. That is, the physical properties and composition of the sample affect the element signal such that changes in one or more of the elements forming the matrix which alter an element signal even though the element concentration remains constant. Matrix effects can be divided into two kinds, physical and chemical. Physical matrix effects depend on the physical properties of the sample and generally relate to the ablation step of LIBS. Major cause of this matrix effects is the change of temperature and electron density of the plasmas induced on different matrix targets which can change the amount of an element ablated. As a result, different excitation and ionization levels of plasma species are achieved. Meanwhile chemical matrix effects occur when the presence of one element affects the emission characteristics of another element. This can complicate calibration of the technique and hence the ability to obtain quantitative results $[8,10]$.

The aim of this study was to demonstrate LIBS technique in detecting calcium content in rice. Twelve sample of rice have been selected for this purpose. The calcium concentration determined by Atomic Absorption Spectroscopy (AAS) technique shows the availability of the element in the sample after it was analyzed using an ungated LIBS system.

\section{EXPERIMENTAL}

Twelve samples of commercial rice were purchased from local markets. The samples offered differences with regard to their rice varieties, grain size, polishing rate and country of origin. List of rice varieties used as sample are shown in Table 1.

Table 1 Varieties of rice used as sample

\begin{tabular}{|c|l|l|}
\hline Sample & Varieties of Rice & Country of Origin \\
\hline A & Local white rice & Malaysia \\
\hline B & Local brown rice & Malaysia \\
\hline C & Imported herbal Ponni rice & India \\
\hline D & Imported glutinous rice & Thailand \\
\hline E & Imported brown rice & Thailand \\
\hline F & Imported Californian rice & USA \\
\hline G & Imported fragrant rice & Thailand \\
\hline H & Local white rice & Malaysia \\
\hline I & Imported parboiled Ponni rice & India \\
\hline J & Imported steamed Ponni rice & India \\
\hline K & Imported Basmathi rice & Pakistan \\
\hline L & Imported fragrant rice & NS \\
\hline NS $=$ & not specified by manufacturer \\
\end{tabular}

Sample preparation for LIBS analysis starts by transforming the sample into pellets. The ground rice samples were sieved before it was made into pellets. Ground rice with the grain size range of less than $100 \mu \mathrm{m}$ was used to be pressed into pellet. Three pellets of each sample were prepared by inserting at about 10 gram of 
sample into the die set with $4 \mathrm{~cm}$ of diameter. The sample in the die set was then applied with $50 \mathrm{kN}$ of force which equivalent to $40 \mathrm{MPa}$ pressure. Holding time is set at 3 minutes. There was no additional binding agent used for pellet making since rice naturally have starch in them. The ground rice and the produced pellet are shown in Figure 2.

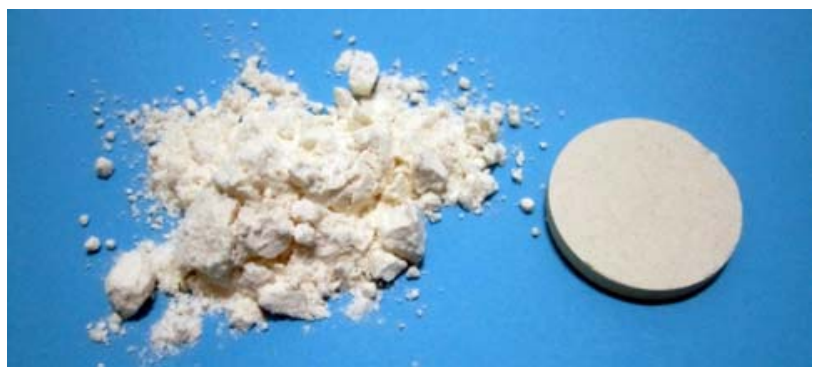

Fig. 2 Ground rice samples were pressed into pellets before LIBS analyses

For the analysis of rice samples using Atomic Absorption Spectroscopy (AAS) teachnique, each sample was digested using nitro-hydrochloric aqua regia. For the digestion of samples, a representative 1 (dry weight) gram of ground sample was digested with repeated additions of nitric acid (HNO3) and hydrochloric acid $(\mathrm{HCl})$. The resultant digestate is filtered and then diluted with deionized water to a final volume of $100 \mathrm{~mL}$ and was analyzed. An atomic absorption spectrometer, model PerkinElmer AAnalyst 400 was used for calcium determinations. Calibration curves for the analysis were done using PerkinElmer Pure Standards. The measurements were performed at $422.67 \mathrm{~nm}$ which correspond to the calcium absorption line respectively. This procedure is to confirm the availability of calcium in all of the selected samples.

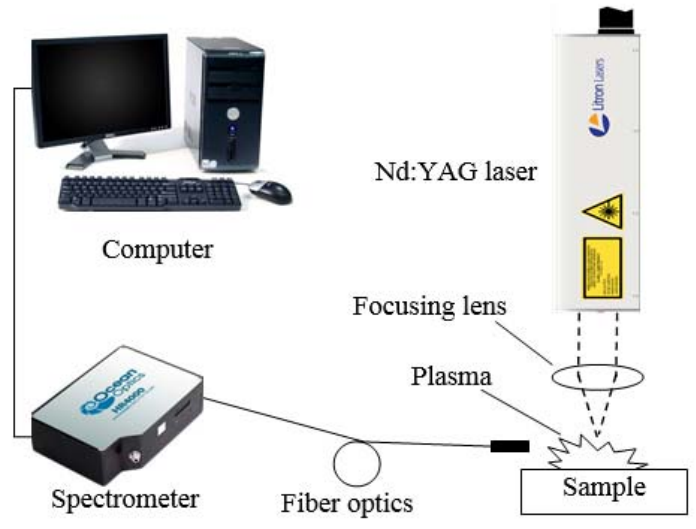

Scheme 1 The schematic diagram of the LIBS system

A schematic diagram of the LIBS system used in this work is shown in Scheme 1. This setup consists of a pulsed laser, fiber optics, focusing lens, a spectrometer and a computer. The Nd:YAG laser which can deliver a maximum single-pulse energy of $60 \mathrm{~mJ}$ with a pulse width of 6-8 ns at a wavelength of $1064 \mathrm{~nm}$ (Nano S 60-30, Litron Lasers Ltd.) was used as an excitation source. In this study, rice samples were analyzed using laser pulse energy of $60 \mathrm{~mJ}$. The laser beam was focused using a focusing lens. The emitted light from plasma was collected by using a collecting lens and was transferred to a spectrometer measuring emission lines from $196.65 \mathrm{~nm}$ to $661.53 \mathrm{~nm}$ with a spectral resolution of $0.12 \mathrm{~nm}$ FWHM (HR4000, Ocean Optics). Around fifty spectra per sample collected which were taken from different regions of each pellet by using High Speed Acquisition technique. All of the collected spectra is analyzed and stored in the computer using SpectraSuite software. The spectra are averaged and plotted using OriginPro 8.5 for baseline removal and peak findings.

\section{RESULTS \& DISCUSSION}

All of the twelve samples have been successfully produced into pellets even without additional binding agent used. Three pellets for each sample have been prepared for LIBS analysis. The surface of the sample is ablated by Nd:YAG laser at fifty different spots. Spectra captured at all fifty different spots are captured by fiber optics which is connected to the spectrometer. All of these spectra are saved in a computer and averaged into one spectrum for each sample. With the exception of a few unidentified lines, LIBS analysis of all rice samples showed that all rice samples have almost the same number of atomic emission lines which represents different elements in the rice samples but different in line intensities.

The ungated LIBS system used for this purpose was able to detect at least seven emission lines from calcium along the wavelength range govern by the spectrometer $(196.65-661.53 \mathrm{~nm})$. In this paper, only two emission lines from calcium will be discussed as both of these peaks gives best signal-to-noise ratio. Figure 3 shows the singly ionized calcium, $\mathrm{Ca}$ II, lines from all of the sample in spectral window ranged between $391 \mathrm{~nm}$ and $398 \mathrm{~nm}$. There are two Ca II lines observed at $393.37 \mathrm{~nm}$ and $396.85 \mathrm{~nm}$ which show high intensity against the baseline. From the averaged spectrum of each sample, different sample shows different intensity on both Ca II lines.

Selecting the right emission line for an element in a LIBS spectrum can be a difficult task since there are many peaks placed closely with each other. The selected peaks can be incorrectly assumed to correspond to the wrong element. In order to confirm these two peaks are in fact singly ionized calcium, Ca II emission lines, intensity ratio calculation of these two peaks is carried out. Intensity ratio of these two peaks - Ca II at 393.37 $\mathrm{nm}$ and $396.85 \mathrm{~nm}$ - has been calculated to confirm these peaks are in fact emission lines from calcium. Intensity ratio is calculated by dividing the intensity of peak at $393.37 \mathrm{~nm}$ over the intensity of peak at $396.85 \mathrm{~nm}$. The 
intensity of both peaks as well as the intensity ratio is plotted as shown in Figure 4. In the entire sample, from sample A to L, intensity of peaks at $393.37 \mathrm{~nm}$ proved to be higher than the intensity of peaks at $396.85 \mathrm{~nm}$. Meanwhile, the calculated intensity ratio between these two peaks remains steady with average of 1.446898 . The intensity ratios on all samples have a quite consistent value even though the intensities of corresponding paired peaks - peaks at 393.37 and $396.85 \mathrm{~nm}$ - are not the same. This relation successfully proved that both of the peaks selected were definitely singly ionized calcium, $\mathrm{Ca}$ II at $393.37 \mathrm{~nm}$ and $396.85 \mathrm{~nm}$.

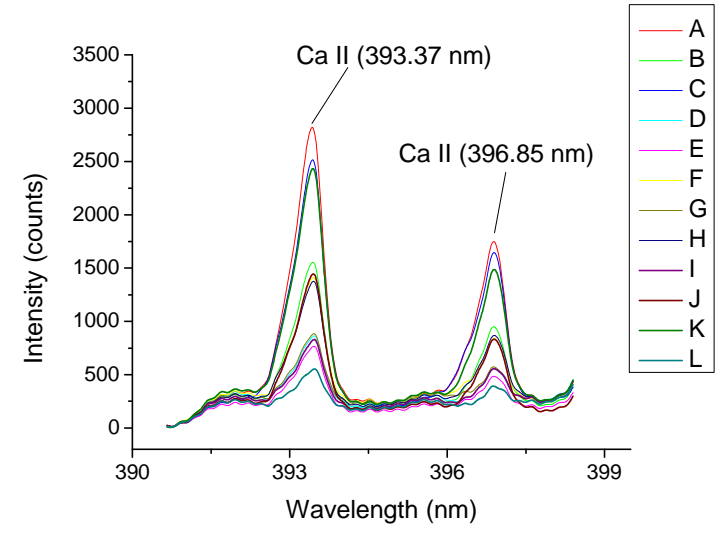

Fig. 3 LIBS spectra of all samples in the spectral range considered for calcium determination which is at $391-398 \mathrm{~nm}$

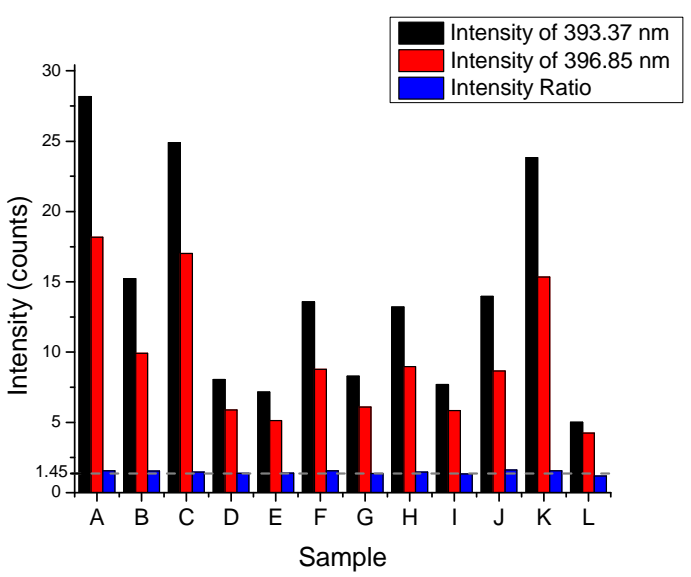

Fig. 4 Intensity ratios of the peaks at $393.36 \mathrm{~nm}$ over $396.85 \mathrm{~nm}$

Further analysis using Atomic Absorption Spectroscopy (AAS) teachnique was done in order to confirm the availability of calcium in all of the sample and also to provide quantitative analysis of calcium concentration. Some manufacturers provide the calcium content of their product. In order to confirm the manufacturer's claim, calcium determination was carried out using AAS. The determined calcium concentration by AAS presented good agreement with the manufacturer's value which shows the availability of the element in the sample. Table 2 shows the calcium concentration in the rice samples determined by AAS and given by the manufacturer. The result shows that calcium is present in the selected samples. Some values appear higher while some are rather lower than the manufacturer's claim. This probably lies in the technique of measurement used by manufacturers to conduct the analysis. Another reasons possibly due to different crops harvested which mainly affected by the soil in which it is grown and the quantity of manure or fertilizers used. Furthermore, loss of nutrient may occur after it is harvested during milling and storage [11]. All of these reasons could explain differences in calcium content in rice sample.

Table 2 Values of calcium determined using AAS

\begin{tabular}{|c|c|c|}
\hline \multirow[t]{2}{*}{ Sample } & \multicolumn{2}{|c|}{ Value of calcium per $100 \mathrm{~g}$ of rice $(\mathrm{mg})$} \\
\hline & Manufacturer & AAS \\
\hline A & 8.00 & 10.12 \\
\hline $\mathrm{B}$ & 15.76 & 20.92 \\
\hline $\mathrm{C}$ & 5.26 & 10.54 \\
\hline $\mathrm{D}$ & NS & 11.74 \\
\hline $\mathrm{E}$ & 16.08 & 7.73 \\
\hline $\mathrm{F}$ & NS & 8.17 \\
\hline $\mathrm{G}$ & 8.00 & 7.90 \\
\hline $\mathrm{H}$ & 6.81 & 5.07 \\
\hline $\mathrm{I}$ & 6.81 & 8.59 \\
\hline $\mathrm{J}$ & NS & 7.92 \\
\hline $\mathrm{K}$ & 5.00 & 7.05 \\
\hline $\mathrm{L}$ & 15.76 & 7.74 \\
\hline
\end{tabular}

$\mathrm{NS}=$ not specified by manufacturer

Sample B have the highest calcium value compared to all of the samples. The calcium value as measured by AAS is $20.92 \mathrm{mg}$ per $100 \mathrm{~g}$ of rice. This sample is from unpolished brown rice which is known to be one of the healthiest varieties of rice. Health conscious consumer should opt for this variety of rice in order to benefits its high calcium content.

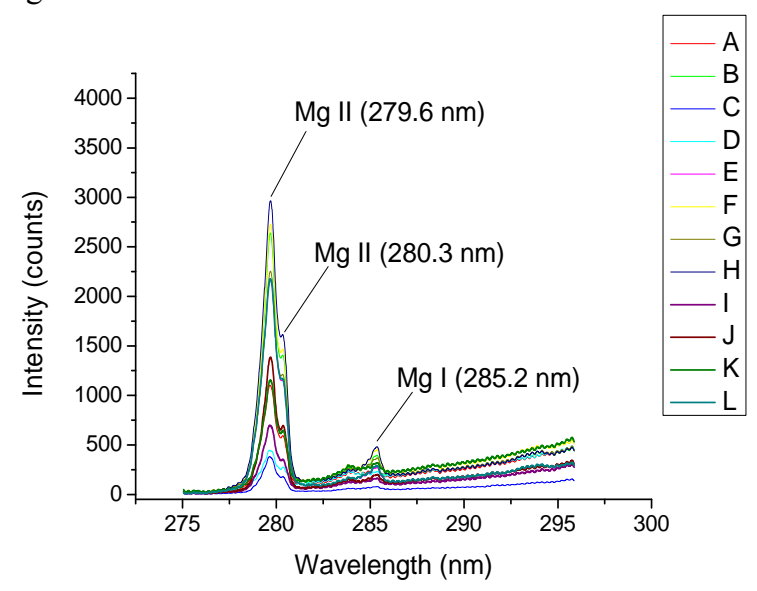

Fig. 5 LIBS spectra of magnesium observed in the rice samples

Apart from calcium lines, there were also magnesium ( $\mathrm{Mg} \mathrm{I}$ and $\mathrm{Mg}$ II) lines observed in Figure 5 with spectral window centered at $285 \mathrm{~nm}$. The high 
intensity spectral lines for singly ionized magnesium, $\mathrm{Mg}$ II at $279.6 \mathrm{~nm}$ and $280.3 \mathrm{~nm}$ were detected in the spectrum. While excited neutral magnesium, $\mathrm{Mg}$ I, line at $285.2 \mathrm{~nm}$ shows lesser intensity which have less significant of signal-to-noise ratio. This results shows that LIBS analysis can also detect other elements simultaneously. The ability to detect more than one element at a time can be favourable compared to other conventional chemical analyses techniques - Atomic Absorption Spectroscopy (AAS) and Atomic Emission Spectroscopy (AES) - as it will reduce time for analysis and the amount of sample required.

The surface of the pellet is captured using a digital microscope and the craters on the surface were measured by using the software provided. Figure 6 shows the surface of sample before and after being ablated by laser for LIBS analysis.
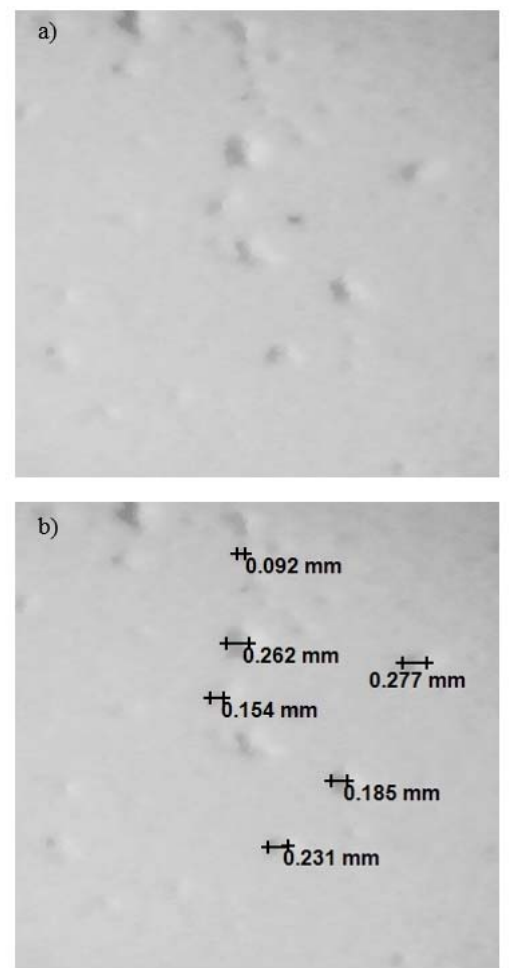

Fig. 6 The surface of the pellet: a) after being ablated by laser, and $b$ ) ablated spot size

The measurement of the ablated surface craters shows different size from one and another. Thus the amount of the sample ablated is also expected to be different with each crater. This can strongly affect LIBS measurement especially when making a calibration curve for quantitative analysis using LIBS system. All of these three factors or a combination of the three could explain the crater irregularities on the sample's surface:

i) Different moisture content in the samples produces different spark intensity. Higher moisture in the sample means more energy from the laser needed for sample ablation. During the sample preparation, there is no procedure to dry the ground rice before it is pressed into pellet. Additional evaluation on the pressed sample regarding its moisture content is needed for verification.

ii) Physical matrix effect depends on the physical properties of the sample such as specific heat and latent heat of vaporization. Meanwhile chemical matrix effects occur when the presence of one element affects the emission characteristics of another element. Different types of rice have different nutrient composition which translates to different chemical matrix effects.

iii) Irregularities on the surface of the sample itself and the effects of shock wave after plasma formation. Shock wave affected the sample surface with the result that the focal spot of the laser is not the same for the following laser pulses. Furthermore, the sample ejected due to the shock wave absorbed incoming laser pulse in front of the sample [12].

Based from these factors, experimental procedures such as sample preparation and LIBS analysis could be refined in order to obtain accurate results in order to do quantitative studies. For the quantitative studies, addition of delay generator to synchronize the laser and spectrometer is important to acquire the best calibration curve. The use of an ablation chamber to control the pressure during laser-material interaction can also increase the quality of LIBS spectra produced. As for qualitative analyses, the results presented in this paper demonstrated high capability of the LIBS system for detecting calcium as well as other elements in rice.

\section{CONCLUSION}

A basic LIBS system was developed to detect calcium in rice using an ungated spectrometer as the detection system. Qualitative analyses of calcium detection in rice are shown practical with LIBS technique. Results show that it can be used to detect other elements simultaneously as well. Quantitative analysis done using AAS showed that sample B - unpolished brown rice have the highest calcium concentration compared to other variety of rice. This work also shows that a LIBS system can be developed without an expensive gated detection system. For quantitative analyses using LIBS, further analyses as well as improvement in the experimental setup are needed to be done.

\section{ACKNOWLEDGEMENT}

The authors acknowledge Universiti Teknologi Malaysia, Faculty of Science and Ministry of Higher Education (MOHE) for supporting this work financially under vote ERGS 4L069 and GUP 03J86.

\section{REFERENCES}

[1] Y. A. Liew, S. R. Syed Omar, M. H. A. Husni, M. A. Z. Abidin, N.A.P. Abdullah, Malaysian Journal of Soil Science Vol. 14, (2010) 71 . 
[2] H. E. Bouis, Field. Crop. Res. 60 (1999) 165.

[3] C. D. Bernaier and J. Zempleni, Micronutrients and Metabolism, CRC Press (2009), p. 111.

[4] W. S. S. Chee, A. R. Suriah, Y. Zaitun, S. P. Chan, S. L. Yap and Y. M. Chan. Asia Pacific J. Clin. Nutr. 11(2), (2002) 142.

[5] N. K. Bhullar \& W. Gruissem, Biotechnol. Adv. (2012).

[6] I. A. H. Ahmad Hanis, S. Jinap, S. Mad Nasir, R. Alias, and A. K. Muhammad Shahrim, International Food Research Journal 19(1) (2012) 363

[7] L. H. Reyes, G. M. M. Rahman and H. M. S. Kingston, Anal. Chim. Acta., 631, (2009).

[8] D. A. Cremers and L. J. Radziemski. History and fundamentals of LIBS, Cambridge University Press, (2006) p. 148.
[9] J. M. Anzano, M. A. Villoria, A. Ru'1z-Medina, R. J. Lasheras. Anal. Chim. Acta. 575 (2006) 230.

[10] E. C. Ferreira, E. A. Menezes, W. O. Matos, D. M. B. P. Milori, A. R. A. Nogueira and L. Martin-Neto, Food Control 21, (2010) 1327.

[11] A. Aadil, M. Shahzad, A. Faiza, K. Ayesha, R. Shakeela and N. Sumera, World Journal of Medical Sciences 6, (2011) 68

[12] D. Daniel, W. H. David and A. Molina, Appl. Spectrosc. 66, (2012) 99. 